\title{
Quantitative evaluation of myocardial fibrosis by cardiac integrated backscatter analysis in Kawasaki disease
}

\author{
Lijian Xie*, Renjian Wang, Min Huang, Yongwei Zhang, Jie Shen and Tingting Xiao
}

\begin{abstract}
Background: Kawasaki disease is an acute, systemic vasculitis that affects the coronary arteries. However, the relationship between myocardial fibrosis and Kawasaki disease has been completely unknown until now. We aimed to provide quantitative information about myocardial fibrosis using cardiac integrated backscatter in Han race Kawasaki disease patients.

Methods: Ninety Kawasaki disease patients and 90 healthy control subjects were recruited. Based on Kawasaki disease status, the patients were categorized into 3 groups: acute, subacute, and convalescence phase. Based on coronary artery status, the Kawasaki disease patients were categorized into 3 groups: without coronary artery lesions, with coronary artery dilation, and with coronary artery aneurysms. All subjects underwent two-dimensional and Doppler examinations to measure clinical echocardiographic parameters. Myocardial fibrosis was detected with calibrated integrated backscatter imaging.

Results: Left ventricle systolic functions were normal in both the Kawasaki disease and control participants. The myocardial calibrated integrated backscatter values of the left ventricles of the acute $(p<0.001)$, subacute $(p<0.001)$ and convalescence phase $(p<0.001)$ Kawasaki disease patients were significantly greater than those of the healthy controls. The left ventricle myocardial calibrated integrated backscatter values were significantly smaller in the Kawasaki disease patients without coronary artery lesions than in the Kawasaki disease patients with coronary artery dilations and coronary artery aneurysms in different phases. The left ventricle myocardial calibrated integrated backscatter results were positively correlated with coronary artery status in the acute $(r=0.331, p<0.001)$, subacute $(r=0.456, p<0.001)$ and convalescence phases $(r=0.407, p<0.001)$ of Kawasaki disease.

Conclusion: Our findings may suggest that myocardial fibrosis occurs during early episodes of Kawasaki disease given uncertainties that exist regarding correlations of calibrated integrated backscatter and myocardial fibrosis.
\end{abstract}

Keywords: Kawasaki disease, Myocardial fibrosis, Cardiac integrated backscatter

\section{Background}

Kawasaki disease is an acute, self-limited, systemic vasculitis of unknown etiology that most notably affects the coronary arteries [1]. The inflammatory process produces peculiar vascular damage that includes coronary artery lesions and myocarditis [2]. Myocardial ischemia remains an important concern, and regular evaluations of myocardial function constitute an important part of the longterm assessment of Kawasaki disease. Some authors have

\footnotetext{
* Correspondence: naijileix@aliyun.com

Department of Cardiology, Shanghai Children's Hospital, Shanghai Jiaotong University, No. 355 Luding Road, Shanghai 200062, China
}

reported that Kawasaki disease patients might be at risk for developing ischemic cardiomyopathy due to abnormal myocardial perfusion and reduced coronary flow reserve, including patients who do not have coronary artery lesions [3-6].

Myocardial fibrosis in Kawasaki disease is still controversial due to limited evidence. Myocardial fibrosis has been documented in one patient via endomyocardial biopsy as long as 11 years after the occurrence of acute Kawasaki disease [7]. Lin et al. [8] found alterations in extracellular matrix biomarkers in Kawasaki disease patients that were suggestive of enhanced collagen synthesis and myocardial fibrosis in adolescents and young adults late after the onset of Kawasaki disease. With 
blood measurement and tissue autopsy, Numano et al. [9] found Galectin-3 could be a marker of myocardial and vascular fibrosis in Kawasaki disease patients with giant aneurysms. However, myocardial fibrosis was not observed in myocardial tissue of Kawasaki disease with normal coronary arteries [9].

However, myocardial biopsies are not practical for myocardial fibrosis diagnoses in children. Furthermore, the cardiac origin of circulating markers of collagen synthesis is difficult to ascertain, and the levels of these markers vary with age and growth, which may confound the interpretation of the results of pediatric studies [10]. In contrast, noninvasive assessment of tissue fibrosis via echocardiographically derived calibrated integrated backscatter has been used in both adults and children [11-13]. Moreover, calibrated integrated backscatter has been used to quantitatively evaluate the echogenicity of the heart in Kawasaki disease [13-16]. Recently, it is questionable about the role of calibrated integrated backscatter as a non-invasive index of fibrosis in clinical studies of patients without extensive fibrosis such as coronary artery disease [17].

However, data derived from small samples, specificities of the equipment used, different measurements of calibrated integrated backscatter and variability due to race may have important effects on calibrated integrated backscatter data. Therefore, the reproducibility of calibrated integrated backscatter measurements needs to be examined in large samples to confirm the reported results. Moreover, whether calibrated integrated backscatter is associated with lesser degrees of fibrosis is unknown. In the present study, we postulated that calibrated integrated backscatter might provide quantitative information about myocardial fibrosis in early episode of Kawasaki disease. Furthermore, we explored the associations of fibrosis with myocardial calibrated integrated backscatter results and the statuses of coronary artery lesions in Kawasaki disease patients.

\section{Methods \\ Subjects}

Ninety Kawasaki disease patients and 90 healthy controls were prospectively recruited from a group of cardiac inpatients and from the clinic (from January 2014 to January 2015). The healthy controls underwent echocardiography examinations for soft heart murmurs in the clinic, and heart disease was completely ruled out for these subjects. The following data were collected from the case notes: demographic data, age at diagnosis, development of coronary artery complications, and current cardiac medications. Based on Kawasaki disease status, the patients were categorized into the following 3 groups: group 1, acute phase (5-10 ${ }^{\text {th }}$ day); group 2 , subacute phase $\left(11-21^{\text {th }}\right.$ day), and group 3 , convalescence phase $\left(28^{\text {th }}\right.$ day $-3^{\text {rd }}$ month).
Group 4 was composed of the healthy controls. Based on coronary artery status, the Kawasaki disease patients were categorized into the following 3 groups: group A, no coronary artery lesions; group B, coronary artery dilation; and group $\mathrm{C}$, coronary artery aneurysms. Group D was composed of the healthy controls. The echocardiographic data were compared between the patients and the healthy agematched control subjects.

Diagnoses of Kawasaki disease were made based on standard clinical criteria [18]. Coronary artery dilations and coronary artery aneurysms were documented by echocardiography according to the criteria set by the Japanese Ministry of Health [19].

\section{Ethics statement}

This study was approved by the Institutional Review Board of Shanghai Children's Hospital/Shanghai Jiaotong University, and informed written consent was obtained from all subjects and parents on behalf of minors.

\section{Clinical echocardiography}

Echocardiography was performed by a single experienced pediatric cardiologist. All the Kawasaki disease patients and controls underwent transthoracic two-dimensional and Doppler examinations using a Philips IE-33 ultrasound machine. The probes used were S4 and S8. The children were prepared for echocardiography via the administration of a sedative (10\% chloral hydrate $50 \mathrm{mg} / \mathrm{kg}$ administered orally) as usually required in clinical practice in China.

\section{Calibrated integrated backscatter assessment}

The calibrated integrated backscatter was assessed using QLAB software. The calibrated integrated backscatter measurements were performed as previously reported [11]. Integrated backscatter curves were acquired in the parasternal short-axis view by locating $3 \times 3$-mm sample volumes in the mid-basal septum, mid-posterior wall, and mid-pericardium in end diastole. The calibrated integrated backscatter measurements were performed at a fixed point in the cardiac cycle (i.e., the peak of the QRS complex) and are expressed in decibels. The pericardial integrated backscatter was used as the baseline. The left ventricular calibrated integrated backscatter was calculated by subtracting the average pericardial integrated backscatter intensity from the average myocardial integrated backscatter intensity of the septum and posterior wall (Fig. 1). The sample volumes were tracked manually to maintain the same region throughout the heart cycle.

\section{Reproducibility}

Off-line analyses of the calibrated integrated backscatter data were performed by two blinded independent observers. Interobserver variability was tested in 10 cases of 


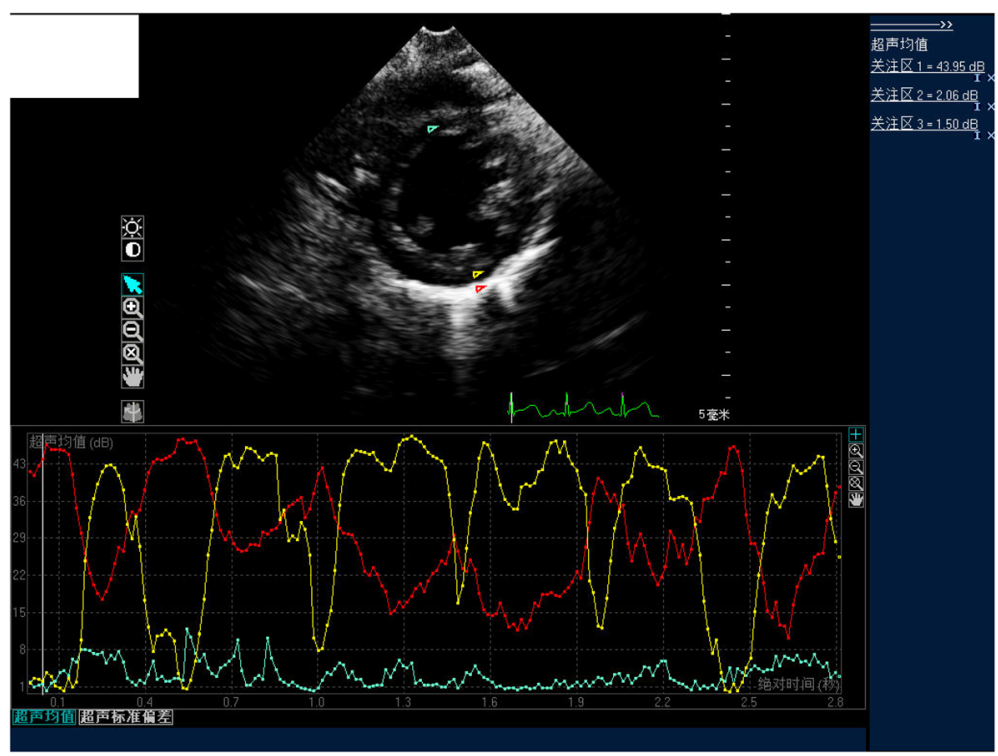

Fig. 1 Calibrated integrated backscatter (CIB) of the left ventricle (LV) assessed with QLAB software

Kawasaki disease and 10 control cases. The same samples were re-evaluated after a 20-day period by one of the two observers to assess intraobserver variability.

\section{Statistical analysis}

All clinical echocardiographic data and integrated backscatter profiles were averaged over three consecutive cardiac cycles. The data are presented as the means \pm the SDs unless otherwise stated. The demographic and echocardiographic parameters were compared between the patients with different Kawasaki disease statuses and the controls using unpaired Student's t tests. The calibrated integrated backscatter values of the patients in the different phases of Kawasaki disease and the healthy controls were compared with one-way ANOVA tests. Tukey's post-hoc test was used whenever statistically significant differences were observed. Spearman correlation analysis was used to determine the association between the myocardial calibrated integrated backscatter results and the coronary artery statuses of the Kawasaki disease patients. P values $<0.05$ were considered statistically significant. All statistical analyses were performed using SPSS version 16.0.

\section{Results}

\section{Subjects}

All subjects were of Han nationality. The 90 (58 males, $64.4 \%)$ Kawasaki disease patients were studied at $18.40 \pm$ 10.07 months, and the 90 (55 males, $61.1 \%$ ) healthy controls were studied at $17.93 \pm 11.59$ months $(p=0.773)$. Neither gender (64.4 \% vs. $61.1 \%, p=0.646)$ nor body weight $(11.72 \pm 3.03 \mathrm{~kg}$ vs. $11.41 \pm 3.48 \mathrm{~kg}, p=0.525)$ differed significantly between the Kawasaki disease and control cohorts.

The gender distribution was different (47 males, $62.7 \%$ vs. 10 males, $83.3 \%$ vs. 1 male, $33.3 \%$ vs. 55 males, $61.1 \%, p<0.001)$ in subgroup A, B, C and D. Neither age $(17.87 \pm 9.74$ vs. $21.92 \pm 11.83$ vs. $17.67 \pm 12.22$ vs. $17.93 \pm$ 11.59 months, $p=0.675)$ nor body weight $(11.63 \pm 2.91 \mathrm{~kg}$ vs. $12.28 \pm 3.70 \mathrm{~kg}$ vs. $11.57 \pm 4.05 \mathrm{~kg}$ vs. $11.41 \pm 3.48 \mathrm{~kg}$, $p=0.846)$ differed significantly in subgroup A, B, C and D.

All patients were treated with $2 \mathrm{~g} / \mathrm{kg}$ immunoglobulin intravenously in a single dose and $50-80 \mathrm{mg} / \mathrm{kg}$ aspirin during the acute phase of the illness $\left(5-10^{\text {th }}\right.$ day) followed by $3-5 \mathrm{mg} / \mathrm{kg}$ per day for $6-8$ weeks after the resolution of the acute illness. Aspirin was continued chronically at the same dose in children in whom coronary abnormalities persisted.

\section{Clinical echocardiographic parameters}

Table 1 summarizes the clinical echocardiographic parameters in patients in the different phases of Kawasaki disease and the control subjects. Left ventricle systolic and diastolic functions were normal, and no significant differences in either parameter were observed between the Kawasaki disease patients and controls. Left ventricle masses did not differ across all of the subjects. Among the Kawasaki disease cohorts, 75 (83.3 \%) cases were without coronary artery lesions, 12 (13.3\%) cases exhibited coronary artery dilation, and 3 (3.3\%) cases had coronary artery aneurysms.

\section{Myocardial calibrated integrated backscatter}

The myocardial calibrated integrated backscatter values for the left ventricle were significantly smaller in the 
Table 1 Clinical echocardiographic parameters in KD and control

\begin{tabular}{|c|c|c|c|c|c|}
\hline & KD (acute) & KD (subacute) & KD (convalescence) & Healthy control & $p$ \\
\hline \multicolumn{6}{|l|}{ Left Ventricle } \\
\hline \multicolumn{6}{|l|}{ M-mode } \\
\hline LV EDD (mm) & $33.6 \pm 3.4$ & $32.9 \pm 3.6$ & $33.3 \pm 3.2$ & $32.7 \pm 2.5$ & 0.56 \\
\hline LV ESD (mm) & $17.1 \pm 1.9$ & $17.6 \pm 1.4$ & $16.9 \pm 2.0$ & $17.2 \pm 1.7$ & 0.32 \\
\hline IVSd (mm) & $4.2 \pm 0.5$ & $4.3 \pm 0.3$ & $4.1 \pm 0.4$ & $4.1 \pm 0.3$ & 0.38 \\
\hline LV PWd (mm) & $4.0 \pm 0.4$ & $4.1 \pm 0.4$ & $4.0 \pm 0.5$ & $4.1 \pm 0.4$ & 0.62 \\
\hline LV mass (g) & $22.9 \pm 0.4$ & $22.3 \pm 0.5$ & $22.8 \pm 0.5$ & $23.0 \pm 0.4$ & 0.33 \\
\hline LV eject fraction (\%) & $68.3 \pm 4.2$ & $70.2 \pm 6.1$ & $69.5 \pm 5.7$ & $68.9 \pm 5.5$ & 0.14 \\
\hline \multicolumn{6}{|l|}{ Mitral inflow velocities } \\
\hline $\mathrm{E}(\mathrm{cm} / \mathrm{s})$ & $97.7 \pm 10.2$ & $105.4 \pm 9.7$ & $93.8 \pm 13.5$ & $98.7 \pm 12.5$ & 0.49 \\
\hline $\mathrm{A}(\mathrm{cm} / \mathrm{s})$ & $59.9 \pm 12.5$ & $57.5 \pm 14.7$ & $61.4 \pm 13.2$ & $62.3 \pm 11.9$ & 0.78 \\
\hline E/A ratio & $1.6 \pm 0.4$ & $1.8 \pm 0.4$ & $1.5 \pm 0.3$ & $1.6 \pm 0.4$ & 0.65 \\
\hline \multicolumn{6}{|l|}{ Right ventricle } \\
\hline \multicolumn{6}{|l|}{ Tricuspid inflow velocities } \\
\hline $\mathrm{E}(\mathrm{cm} / \mathrm{s})$ & $71.0 \pm 12.1$ & $74.3 \pm 10.5$ & $69.8 \pm 8.4$ & $73.4 \pm 15.1$ & 0.84 \\
\hline $\mathrm{A}(\mathrm{cm} / \mathrm{s})$ & $50.0 \pm 6.9$ & $54.9 \pm 9.8$ & $53.8 \pm 7.3$ & $51.5 \pm 5.3$ & 0.33 \\
\hline E/A ratio & $1.4 \pm 0.3$ & $1.4 \pm 0.2$ & $1.3 \pm 0.2$ & $1.4 \pm 0.3$ & 0.79 \\
\hline
\end{tabular}

Abbreviations: KD Kawasaki disease, $L V$ left ventricle, EDD end-diastolic dimension, ESD end-systolic dimension, IVSd interventricular septal thickness at diastole, $P W d$ posterior wall thickness at diastole

healthy control subjects than the Kawasaki disease patients in the acute $(-22.57 \pm 5.43$ vs. $-17.60 \pm 4.78, p<0.001)$, subacute $(-22.57 \pm 5.43$ vs. $-18.31 \pm 4.27, p<0.001)$ and convalescence $(-22.57 \pm 5.43$ vs. $-18.71 \pm 4.30, p<0.001)$ phases (Fig. 2). Furthermore, the myocardial calibrated integrated backscatter values for the left ventricle were significantly smaller in the healthy controls than in the Kawasaki disease patients without coronary artery lesions in the acute $(-22.57 \pm 5.43$ vs. $-18.93 \pm 3.95, \quad p<0.001), \quad$ subacute $(-22.57 \pm 5.43$ vs. $-19.51 \pm 3.50, p<0.001)$ and convalescence $(-22.57 \pm 5.43$ vs. $-19.98 \pm 3.36, p<0.001)$ phases

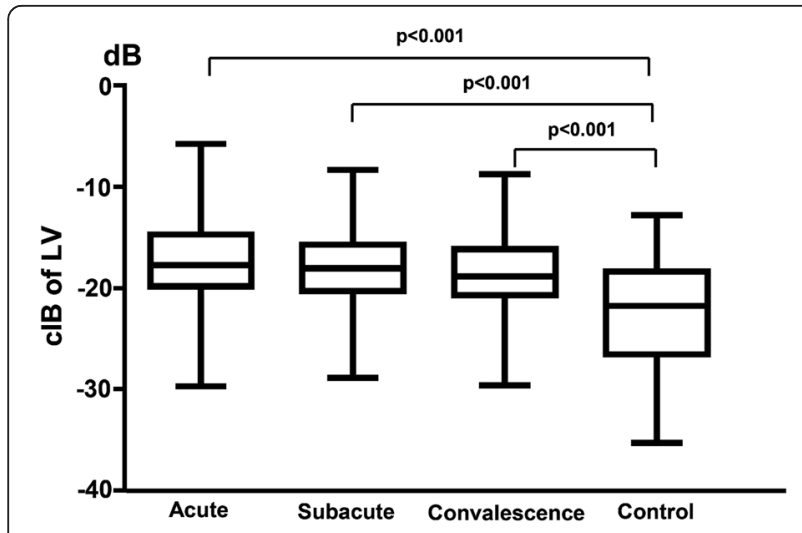

Fig. 2 Bar graph illustrating the calibrated integrated backscatter (CIB) values of the left ventricles (LVs) in different phases of KD and in healthy control subjects
(Fig. 3). Finally, the myocardial calibrated integrated backscatter values for the left ventricle were significantly greater in the Kawasaki disease patients with coronary artery aneurysms and coronary artery dilation compared with the Kawasaki disease patients without coronary artery lesions in the acute $(-7.77 \pm 1.71$ vs. $-11.71 \pm 1.85$ vs. $-18.93 \pm 3.95)$, subacute $(-9.27 \pm 0.85$ vs. $-13.08 \pm 1.85$ vs. $-19.51 \pm 3.5)$ and convalescence phase $(-9.23 \pm 0.31$ vs. $-13.13 \pm 2.02$ vs. $-19.98 \pm 3.36$ ) of illness (Fig. 4).

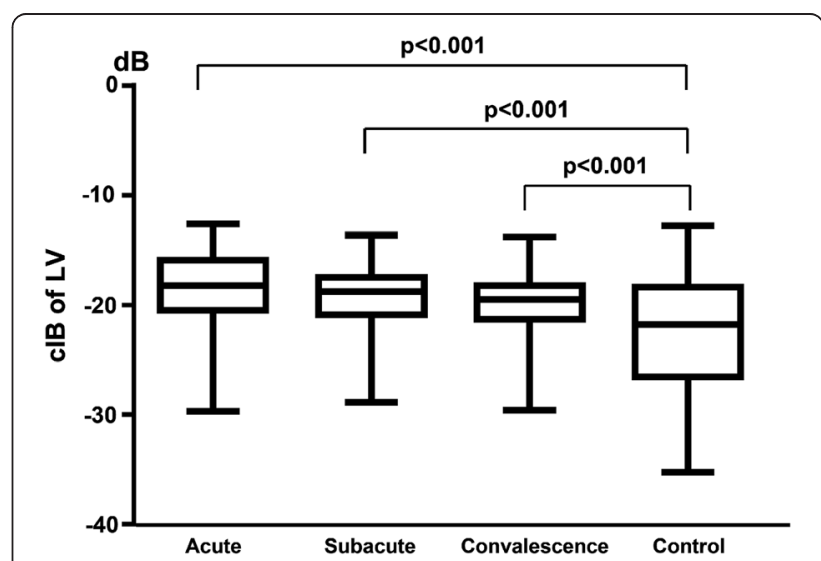

Fig. 3 Bar graph illustrating the calibrated integrated backscatter (ClB) values of the left ventricles (LVs) in different phases of KD without coronary artery lesions (CALs) and in healthy control subjects 


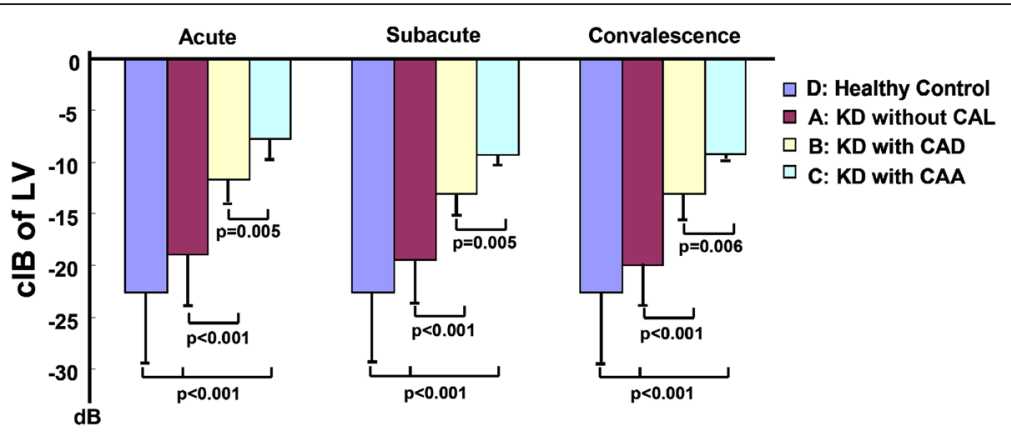

Fig. 4 Histogram illustrating the calibrated integrated backscatter (cIB) values for the left ventricles (LV) in the different KD phase cohorts with and without coronary artery lesions (CALS). CAD: coronary artery dilation; CAA: coronary artery aneurysm

\section{Correlates of calibrated integrated backscatter}

Among the cohort of patients, the average myocardial calibrated integrated backscatter values were positively correlated with the Kawasaki disease patients' coronary artery statuses in the acute $(r=0.331, p<0.001)$, subacute $(r=0.456, p<0.001)$ and convalescence $(r=0.407$, $p<0.001$ ) phases (Fig. 5).

\section{Reproducibility}

The intra- and interobserver variability for the calibrated integrated backscatter observations was $1.6 \pm 1.5$ (correlation was 0.89 (95\% CI 0.75 to $0.95, p<0.001)$ ), and $1.7 \pm$ 1.3 (correlation was 0.83 (95\% CI 0.70 to $0.89, p<0.001)$ ), respectively.

\section{Discussion}

The present study demonstrated that increased myocardial calibrated integrated backscatter values are suggestive of myocardial fibrosis in Kawasaki disease patients with and without coronary artery lesions despite similar measures of left ventricle function between the Kawasaki disease patients and healthy controls. Additionally, the myocardial calibrated integrated backscatter results were found to be positively associated with the Kawasaki disease patients' coronary artery statuses.

Calibrated integrated backscatter is the intensity of an ultrasound wave (scattering) within a particular frequency range, as reflected by a tissue interface that is smaller than the ultrasound wavelength ( $500 \mu \mathrm{m}$ for a $3-\mathrm{MHz}$ probe); thus, the structures that contribute to the calibrated integrated backscatter signal should be 100-125 $\mu \mathrm{m}$ or smaller [20]. Myofibers, extracellular matrix collagen, mitochondria, and small vessels are thought to be the principal backscatterers in the myocardium [21]. With the development of echocardiolographic technologies, the relationship between calibrated integrated backscatter and myocardial fibrosis has been confirmed in human disease [12, 22-24].

Kawasaki disease is a systemic vasculitis of unknown origin that occurs primarily in children younger than
5 years. Many studies have focused on Kawasaki disease with coronary artery lesions. However, few pathological studies have examined the after-effects of Kawasaki disease on the myocardium based on endomyocardial biopsies [7, 25]. Recently, Yonesaka et al. [26] found that the incidence of histopathological abnormalities, such as degeneration, hypertrophy, and inflammatory cell infiltration, are quite high upon initial study and that inflammatory cell infiltration, interstitial fibrosis, and disarray were very noticeable upon follow-up biopsies in Kawasaki disease with giant coronary artery aneurysm. Furthermore, a previous clinical study provided evidence of altered myocardial extracellular matrix turnover with a possible predisposition to increased interstitial fibrosis in adults with Kawasaki disease histories [8]. Leonardi et al. [13] confirmed a significant increase in myocardial calibrated integrated backscatter values in Kawasaki disease patients at least 1 year after the acute episode. Nagata et al. [14] reported that the integrated backscatter values of the coronary artery wall, mitral valve, papillary muscle and ascending aortic wall in Kawasaki disease patients in the acute phase are significantly higher than those of control subjects. Abe et al. [15] also reported that the integrated backscatter values for the coronary artery wall are higher in Kawasaki disease patients than in controls. Moreover, these authors also reported that the integrated backscatter values for the coronary artery wall are higher in pre-immunoglobulin therapy patients than post-immunoglobulin therapy patients with Kawasaki disease. However, Yu et al. [16] used a similar method and reported no significant difference in the perivascular echo brightness of the coronary arteries between Kawasaki disease patients.

The numbers of Kawasaki disease and control subjects are much greater in the present study than those in previous reports. However, the present study is the first to report on the echogenicity of the left ventricle myocardium in Han race Kawasaki disease patients based on the calibrated integrated backscatter method with QLAB software. Our results demonstrated greater 

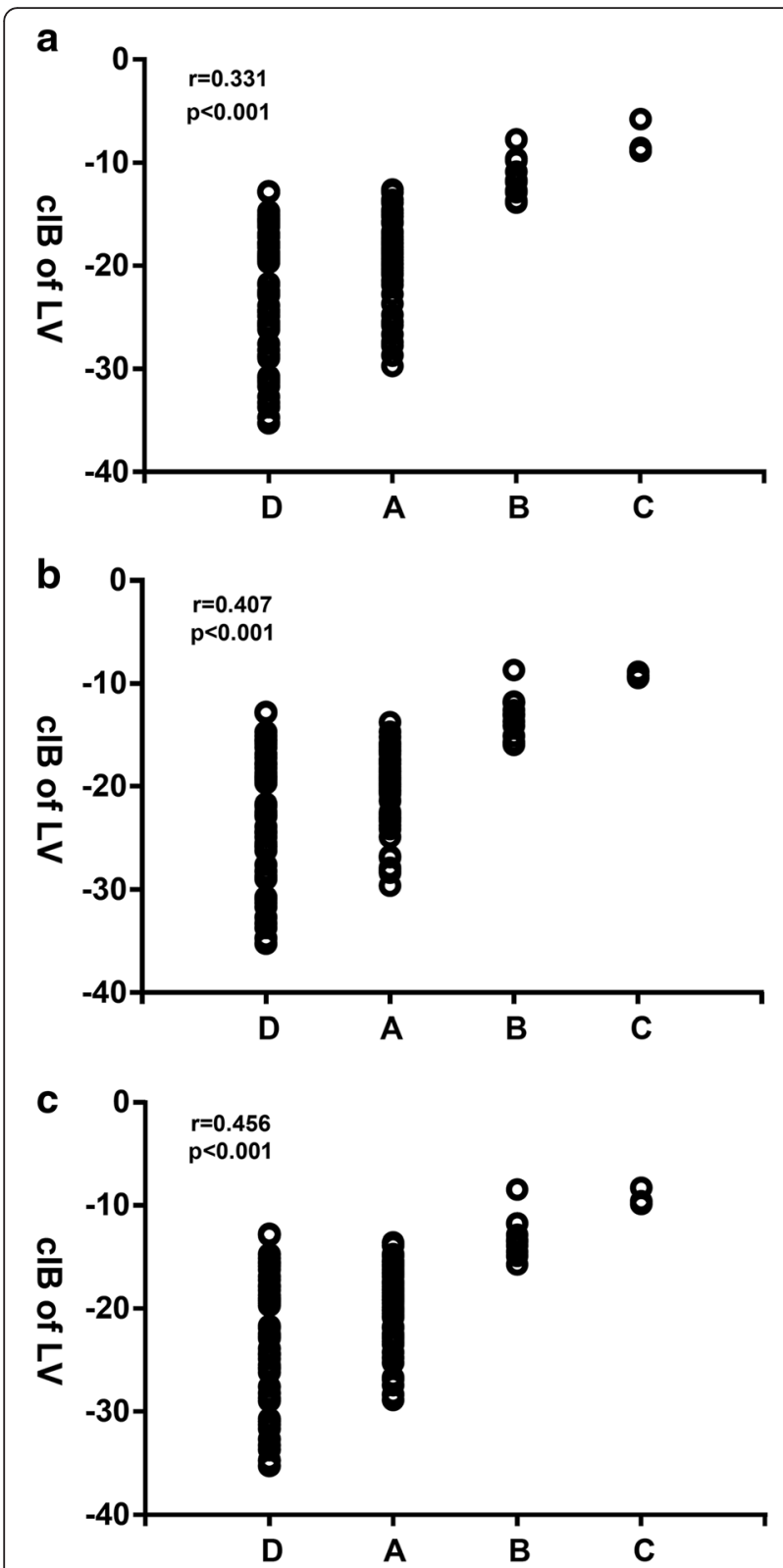

Fig. 5 Scatter plots illustrating the correlations between the myocardial calibrated integrated backscatter values and coronary artery statuses in (a) the acute phase, (b) the subacute phase, and (c) the convalescence phase. D: healthy controls; $A$ : KD without $C A L ; B$ : KD with CAD; C: KD with CAA

myocardial calibrated integrated backscatter values in the Kawasaki disease patients in the acute, subacute and convalescence phases compared with controls. Moreover, greater myocardial calibrated integrated backscatter values observed in the Kawasaki disease patients with coronary artery lesions compared with the Kawasaki disease patients without coronary artery lesions. Finally, we revealed that the calibrated integrated backscatter values were positively correlated with the Kawasaki disease patients' coronary artery statuses.
Calibrated integrated backscatter data have been correlated with myocardial collagen distribution and content and are thought to be a marker of fibrosis [12, 13]. Myocarditis is uniformly present during the acute phase of Kawasaki disease and resolves early in its clinical course. An increase in collagen content is a common response to myocarditis and might have been the cause of the elevated calibrated integrated backscatter values observed in our patients. Thus, histological alterations, such as edema and cell infiltration associated with inflammation of tissues, alter the acoustic properties of tissues [27]. The results of the present study suggest that edematous changes and cell infiltration in the ventricular myocardium will develop in the acute phase of Kawasaki disease and persist for a long time. Our data indicate that myocardial fibrosis may occur during early episodes of Kawasaki disease and that calibrated integrated backscatter is a simple, easy, and noninvasive echocardiographic method for the evaluation of myocardial fibrosis in Kawasaki disease.

Several limitations to this study warrant discussion. First, the subjects' thoracic thicknesses may have affected the calibrated integrated backscatter values, but we did not consider this issue. Second, the modality of cardiac magnetic resonance imaging (MRI) T1 mapping, which is ideal for assessments of diffuse myocardial fibrosis, was not available to us due to economic reasons and the requirement for deeper sedation. Notably, however, conditions other than fibrosis may also influence extracellular volume measurements acquired via $\mathrm{T} 1$ mapping [28]. Third, we did not examine circulating markers of collagen synthesis in the present study. The cardiac origins of these collagen synthesis biomarkers are difficult to ascertain. More importantly, the levels of these markers vary with age and growth, which may confound the interpretation of the results of pediatric studies [10]. Fourth, lack of histological evidence, we could not evaluate the extensive myocardial fibrosis in Kawasaki disease. So, calibrated integrated backscatter maybe reflect not only fibrosis but also myocardium inflammation especially in acute phase. Finally, we did not examine the LV strains or strain rates with tissue Doppler or two-dimensional echocardiography in the present study.

\section{Conclusion}

Our findings suggest that calibrated integrated backscatter values are different in Kawasaki disease and may suggest myocardial fibrosis occurs during early episodes of Kawasaki disease given uncertainties that exist regarding correlations of calibrated integrated backscatter and myocardial fibrosis. Furthermore, calibrated integrated backscatter value is related to the status of the coronary artery in Kawasaki disease. Our findings reinforce the 
concept that long-term follow-up is appropriate for all Kawasaki disease patients independent of the presence of coronary artery lesions. Whether anti-fibrotic strategies, such as aldosterone antagonism, may alter myocardial acoustic properties and improve myocardial fibrosis progression in Kawasaki disease is a topic for further studies.

\section{Competing interests}

The authors declare that they have no competing interest.

\section{Authors' contributions}

LX analyzed and interpreted the subjects' data and written the paper. RJW $\mathrm{MH}$ and YWZ collected the patient data. LX, TTX and JS performed the examination of the subjects' echocardiography. All authors read and approved the final manuscript.

\section{Acknowledgements}

The authors would like to thank Dr. Jian Zhao for his clinical data collection.

\section{Financial Support}

This research received grants from Shanghai Jiaotong University Medical and Engineer Cross Funding (grant number: YG2013MS73), Shanghai Healthy Committee Funding (grant number: 201540099) and Appropriate Technology of Shanghai Municipal Hospital Funding (grant number: SHDC12012238)

Received: 3 October 2015 Accepted: 6 January 2016

Published online: 12 January 2016

\section{References}

1. Kawasaki T, Kosaki F, Okawa S, Shigematsu I, Yanagawa H. A new infantile acute febrile mucocutaneous lymph node syndrome prevailing in Japan. Pediatrics. 1974:54:271-6.

2. Melish ME. Kawasaki syndrome. Pediatr Rev. 1996;17:153-62.

3. Muzik O, Paridon SM, Singh TP, Morrow WR, Dayanikli F, Di Carli MF. Quantification of myocardial blood flow and flow reserve in children with a history of Kawasaki disease and normal coronary arteries using positron emission tomography. J Am Coll Cardiol. 1996;28:757-62.

4. Furuyama $H$, Odagawa $Y$, Katoh $C$, Iwado $Y$, Yoshinaga $K$, Ito $Y$, et al. Assessment of coronary function in children with a history of Kawasaki disease using (15) O-water positron emission tomography. Circulation. 2002; 105:2878-84.

5. Paridon SM, Galioto FM, Vincent JA, Tomassoni TL, Sullivan NM, Bricker JT. Exercise capacity and incidence of myocardial perfusion defects after Kawasaki disease in children and adolescents. J Am Coll Cardiol. 1995;25:1420-4.

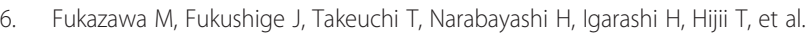
Discordance between thallium-201 scintigraphy and coronary angiography in patients with Kawasaki disease: myocardial ischemia with normal coronary angiogram. Pediatr Cardiol. 1993;14:67-74.

7. Yutani C, Go S, Kamiya T, Hirose O, Misawa H, Maeda H, et al. Cardiac biopsy of Kawasaki disease. Arch Pathol Lab Med. 1981;105:470-3.

8. Lin MT, Chen SJ, Ho YL, Huang KC, Chen CA, Chiu SN, et al. Abnormal matrix remodeling in adolescents and young adults with Kawasaki disease late after onset. Clinical Chemistry. 2008;54:1815-22.

9. Numano F, Shimizu C, Jimenez-Fernandez S, Vejar M, Oharaseki T, Takahashi K, et al. Galectin-3 is a marker of myocardial and vascular fibrosis in Kawasaki disease patients with giant aneurysms. Int J Cardiol. 2015;201:429-37.

10. Trivedi P, Risteli J, Risteli L, Hindmarsh PC, Brook CG, Mowat AP. Serum concentrations of the type I and III procollagen propeptides as biochemical markers of growth velocity in healthy infants and children and in children with growth disorders. Pediatr Res. 1991;30(3):276-80.

11. Pérez JE, Miller JG, Holland MR, Wickline SA, Waggoner AD, Barzilai B, et al. Ultrasonic tissue characterization: integrated backscatter imaging for detecting myocardial structural properties and on-line quantitation of cardiac function. Am J Card Imaging. 1994;8:106-12.

12. Kosmala W, Przewlocka-Kosmala M, Wojnalowicz A, Mysiak A, Marwick TH. Integrated backscatter as a fibrosis marker in the metabolic syndrome: association with biochemical evidence of fibrosis and left ventricular dysfunction. Eur Heart J Cardiovasc Imaging. 2012;13:459-67.

13. Leonardi B, Giglio V, Sanders SP, Pasceri V, De Zorzi A. Ultrasound tissue characterization of the myocardium in patients after Kawasaki disease. Pediatr Cardiol. 2010;31:766-72.

14. Nagata H, Yamamura K, Uike K, Nakashima Y, Hirata Y, Morihana E, et al. Evaluation of echogenicity of the heart in Kawasaki disease. Eur J Pediatr. 2014;173:1089-93.

15. Abe O, Karasawa K, Hirano M, Miyashita M, Taniguchi K, Ayusawa M, et al. Quantitative evaluation of coronary artery wall echogenicity by integrated backscatter analysis in Kawasaki disease. J Am Soc Echocardiogr. 2010;23:938-42.

16. Yu JJ, Jang WS, Ko HK, Han MK, Kim YH, Ko JK, et al. Perivascular brightness of coronary arteries in Kawasaki disease. J Pediatr. 2011;159:454-7.

17. Prior DL, Somaratne JB, Jenkins AJ, Yii M, Newcomb AE, Schalkwijk CG, et al. Calibrated integrated backscatter and myocardial fibrosis in patients undergoing cardiac surgery. Open Heart. 2015;2(1), e000278.

18. Newburger JW, Takahashi M, Gerber MA, Han MK, Kim YH, Ko JK, et al. Committee on rheumatic fever, endocarditis and Kawasaki disease, council on cardiovascular disease in the young, American heart association, American academy of pediatrics. Diagnosis, treatment, and long-term management of Kawasaki disease: a statement for health professionals from the committee on rheumatic fever, endocarditis and Kawasaki disease, council on cardiovascular disease in the young, American heart association. Circulation. 2004:110:2747-71.

19. Research Committee on Kawasaki Disease. Report of Subcommittee on standardization of diagnostic criteria and reporting of coronary artery lesion in Kawasaki disease. Japan, Ministry of Health and Welfare: Tokyo; 1984. p. 55-66.

20. Rhyne TL, Sagar KB. IBR5: an optimal measurement of integrated backscatter and cyclic variation of integrated backscatter. Ultrason Imaging. 1990;12:189-204.

21. Gong L, Wang ZG, Ran HT, Ling ZY, Tang HL, Zheng YY, et al. Relationship between myocardial ultrasonic integrated backscatter and mitochondria of the myocardium in dogs. Clin Imaging. 2006:30:402-8.

22. Kosmala W, Przewlocka-Kosmala M, Szczepanik-Osadnik H, Mysiak A, Marwick TH. Fibrosis and cardiac function in obesity: a randomised controlled trial of aldosterone blockade. Heart. 2013;99:320-6.

23. Bertini M, Delgado V, den Uijl DW, Nucifora G, Ng ACT, van Bommel RJ, et al. Prediction of cardiac resynchronization therapy response: value of calibrated integrated backscatter imaging. Circ Cardiovasc Imaging. 2010;3:86-93.

24. Maceira AM, Barba J, Varo N, Beloqui O, Díez J. Ultrasonic backscatter and serum marker of cardiac fibrosis in hypertensives. Hypertension. 2002;39:923-8.

25. Yonesaka S, Takahashi T, Matubara T, Nakada T, Furukawa H, Tomimoto K, et al. Histopathological study on Kawasaki disease with special reference to the relation between the myocardial sequelae and regional wall motion abnormalities of the left ventricle. Jpn Circ J. 1992;56:352-8.

26. Yonesaka S, Takahashi T, Eto S, Sato T, Otani K, Ueda T, et al. Biopsy-proven myocardial sequels in Kawasaki disease with giant coronary aneurysms. Cardiology in the Young. 2010;20:602-9.

27. Lieback E, Hardouin I, Meyer R, Bellach J, Hetzer R. Clinical value of echocardiographic tissue characterization in the diagnosis of myocarditis. Eur Heart J. 1996;17:135-42.

28. Shah RV, Abbasi SA, Neilan TG, Hulten E, Coelho-Filho O, Hoppin A, et al. Myocardial tissue remodeling in adolescent obesity. J Am Heart Assoc. 2013;2, e000279.

\section{Submit your next manuscript to BioMed Central} and we will help you at every step:

- We accept pre-submission inquiries

- Our selector tool helps you to find the most relevant journal

- We provide round the clock customer support

- Convenient online submission

- Thorough peer review

- Inclusion in PubMed and all major indexing services

- Maximum visibility for your research

Submit your manuscript at www.biomedcentral.com/submit
) Biomed Central 